\title{
Update on the hadronic vacuum polarisation contributions to muon $g-2$ and $\alpha\left(\mathbf{m}_{\mathrm{Z}}^{2}\right)$
}

\author{
Zhiqing Zhang ${ }^{1, \star}$ \\ ${ }^{1}$ Laboratoire de l'Accélérateur Linéaire, IN2P3-CNRS et Université Paris-Sud 11, F-91898, Orsay Cedex, \\ France
}

\begin{abstract}
An update of the hadronic vacuum polarisation contributions to the muon magnetic anomaly and to the running of the electromagnetic couplings constant at the $Z$-boson mass is presented. Newest $e^{+} e^{-} \rightarrow$ hadrons cross-section data mainly from the BABAR and VEPP-2000 experiments have been included. For the muon $(g-2) / 2$, the lowest-order hadronic contribution is evaluated to be $(693.1 \pm 3.4) \cdot 10^{-10}$, improving the precision of our previous evaluation by $21 \%$. The full Standard Model prediction differs by $3.5 \sigma$ from the experimental value. The five-quark hadronic contribution to $\alpha\left(m_{Z}^{2}\right)$ is evaluated to be $(276.0 \pm 0.9) \cdot 10^{-4}$.
\end{abstract}

\section{Introduction}

The Standard Model (SM) predictions of the anomalous magnetic moment of the muon, $a_{\mu}=$ $\left(g_{\mu}-2\right) / 2$, with $g_{\mu}$ the muon gyromagnetic factor, and of the running electromagnetic coupling constant, $\alpha(s)$, a crucial ingredient of electroweak theory, are limited in precision by hadronic vacuum polarisation (HVP) contributions. The dominant hadronic terms can be calculated with a combination of experimental cross-section data, involving $e^{+} e^{-}$annihilation to hadrons, and perturbative QCD. They are used to evaluate energy-squared dispersion integrals ranging from the $\pi^{0} \gamma$ threshold to infinity. The kernels occurring in these integrals emphasise low photon virtuality, owing to the $1 / \mathrm{s}$ descent of the cross-section, and, in case of $a_{\mu}$, to an additional $1 / s$ suppression. In the latter case, about $74 \%$ of the lowest order hadronic contribution and $59 \%$ of the total uncertainty-squared are given by the $\pi^{+} \pi^{-}(\gamma)$ final state, while this channel amounts to only $12 \%$ of the hadronic contribution to $\alpha(s)$ at $s=m_{Z}^{2}$.

In this proceedings in common with that of EPS 2017, we report an update, detailed in Ref. [1], of the lowest-order hadronic contribution, $a_{\mu}^{\text {had,LO }}$, to the muon magnetic anomaly, and the hadronic contribution, $\Delta \alpha_{\text {had }}\left(m_{Z}^{2}\right)$, to $\alpha(s)$ at the $Z$-boson mass using newest $e^{+} e^{-} \rightarrow$ hadrons cross-section data.

\section{Updated results}

Exclusive bare hadronic cross-section measurements are integrated up to $1.8 \mathrm{GeV}$ over the relevant dispersion kernels using the software package HVPTools [2]. In this update, 39 channels are included,

^ in collaboration with M. Davier, A. Hoecker and B. Malaescu. e-mail: zhangzq@1al.in2p3.fr 
Table 1. Comparison of the new evaluation with our previous one and their relative uncertainty reduction. Where three (or more) uncertainties are given, the first is statistical, the second channel-specific systematic, and

the third common systematic, which is correlated with at least one another channel. For the contribution computed from QCD, only the total uncertainties are given, which include effects from the $\alpha_{s}$ uncertainty, the truncation of the perturbative series at four loops, the FOPT vs. CIPT ambiguity, and quark mass uncertainties. The additional uncertainty dubbed "dual" estimates possible quark-hadron duality violating effects in the QCD estimate between 1.8 and $2.0 \mathrm{GeV}$.

\begin{tabular}{lrrr}
\hline Channel & $a_{\mu}^{\text {had,LO }}\left[10^{-10}\right] 2017$ & $a_{\mu}^{\text {had,LO }}\left[10^{-10}\right] 2011$ & $\delta a_{\mu}^{\text {had,LO }}$ reduction \\
\hline$\pi^{+} \pi^{-}$ & $507.14 \pm 1.13 \pm 2.20 \pm 0.75$ & $507.80 \pm 1.22 \pm 2.50 \pm 0.56$ & $-9 \%$ \\
$2 \pi^{+} 2 \pi^{-}$ & $13.68 \pm 0.03 \pm 0.27 \pm 0.14$ & $13.35 \pm 0.10 \pm 0.43 \pm 0.29$ & $-42 \%$ \\
$\pi^{+} \pi^{-} 2 \pi^{0}$ & $18.03 \pm 0.06 \pm 0.48 \pm 0.26$ & $18.01 \pm 0.14 \pm 1.17 \pm 0.40$ & $-56 \%$ \\
$K^{+} K^{-}$ & $22.81 \pm 0.24 \pm 0.28 \pm 0.17$ & $21.63 \pm 0.27 \pm 0.58 \pm 0.36$ & $-46 \%$ \\
$K_{S}^{0} K_{L}^{0}$ & $12.82 \pm 0.06 \pm 0.18 \pm 0.15$ & $12.96 \pm 0.18 \pm 0.25 \pm 0.24$ & $-38 \%$ \\
$K \bar{K} \pi$ & $2.45 \pm 0.06 \pm 0.12 \pm 0.07$ & $(2.39 \pm 0.07 \pm 0.12 \pm 0.08)$ & $-6 \%$ \\
$K \bar{K} 2 \pi$ & $0.85 \pm 0.02 \pm 0.05 \pm 0.01$ & $(1.35 \pm 0.09 \pm 0.38 \pm 0.03)$ & $-86 \%$ \\
Missing $(\%)$ & $0.09 \pm 0.02$ & $0.69 \pm 0.07$ & \\
$R_{\text {QCD }}[1.8-3.7 \mathrm{GeV}]_{u d s}$ & $33.45 \pm 0.28 \pm 0.59_{\text {dual }}$ & $33.45 \pm 0.28$ & \\
Sum & $693.1 \pm 1.2 \pm 2.6 \pm 1.7 \pm 0.1 \Psi \pm 0.7_{\text {QCD }}$ & $692.3 \pm 1.4 \pm 3.1 \pm 2.4 \pm 0.2 \pm 0.3$ & $-21 \%$ \\
\hline
\end{tabular}

as compared to only 22 in our previous work from 2011 [3]. Channels having important improvements (changes) are shown in Table 1.

For the dominant $\pi^{+} \pi^{-}$channel, the computation of the dispersion integral over the full $\pi^{+} \pi^{-}$ spectrum requires to extend the available data to the region between threshold and $0.3 \mathrm{GeV}$, for which a fit as described in Ref. [2] is used. A tension between the BABAR and KLOE measurements is observed at the above the $\rho(770)$ peak region [1]. The local uncertainty rescaling applied increased the combined $a_{\mu}^{\text {had,LO }}$ uncertainty by $15 \%$ in the channel. In spite of this problem, the precision of the $\pi^{+} \pi^{-}$channel of the new reevaluation is improved by $9 \%$, which includes recent measurements from KLOE-2012 [4] and BESIII-2015 [5].

The $2 \pi^{+} 2 \pi^{-}$channel includes an updated measurement from BABAR-2012 [6] using its full available data sample and a new measurement from CMD3-2017 [7]. The total uncertainty of 0.31 (in units of $10^{-10}$ unless otherwise specified) on the corresponding combined HVP contribution is reduced by $-42 \%$. The precision of the $\pi^{+} \pi^{-} 2 \pi^{0}$ channel is improved by $56 \%$ with the final BABAR-2017 data [8] that have its systematic uncertainty reduced to $3.1 \%$ below $2.7 \mathrm{GeV}$ from about $10 \%$ in its preliminary version.

Including new measurements from BABAR-2013 [9] and SND-2016 [10] reduces the uncertainty of the $K^{+} K^{-}$channel by $-46 \%$. However both data, in agreement with each other, show a discrepancy with the former SND data that exceeds the quoted systematic uncertainty. The $K_{S}^{0} K_{L}^{0}$ channel has received two new data sets from BABAR-2014 [11] and CMD3-2016 [12]. The precision of this channel is improved by $38 \%$.

In previous hadronic polarisation analyses the available exclusive $e^{+} e^{-} \rightarrow K \bar{K}+$ pions data were incomplete. Missing channels were constrained based on assumptions about the process dynamics and isospin symmetry [3] leading to considerable uncertainty. This procedure became unnecessary since the BABAR experiment produced cross-section results for three channels contributing to the final state $K \bar{K} \pi$ and six channels contributing to $K \bar{K} \pi \pi$. This together with many processes with smaller cross sections measured from VEP-2000 and BABAR explains the improvements for the $K \bar{K} \pi, K \bar{K} \pi \pi$ and the missing channel in Table 1.

The continuum contribution in the non-resonant energy ranges above $1.8 \mathrm{GeV}$ is calculated using four-loop perturbative QCD including $O\left(\alpha_{s}^{2}\right)$ quark mass corrections. In the energy range of 
$1.8-3.7 \mathrm{GeV}$, an uncertainty of 0.59 is added. This corresponds to the full difference between the perturbative calculation and the sum of exclusive measurements in the narrow energy interval $1.8-2.0 \mathrm{GeV}$. It accounts for possible low-mass quark-hadron duality violation affecting the perturbative QCD approximation.

The sum of all lowest-order hadronic contributions, $a_{\mu}^{\text {had, } \mathrm{LO}}=691 \pm 3.4$, is improved by $21 \%$ in precision and is still dominated by experimental systematic uncertainties. Including the contributions from higher order hadronic loops, $-9.87 \pm 0.09$ (NLO) and $1.24 \pm 0.01$ (NNLO) [13], hadronic lightby-light scattering, $10.5 \pm 2.6$ [14], as well as QED, $11658471.895 \pm 0.008$ [15], and electroweak effects, $15.36 \pm 0.10$ [16], the complete SM prediction amounts to, $a_{\mu}^{\mathrm{SM}}=11659182.3 \pm 3.4 \pm$ $2.6 \pm 0.2\left(4.3_{\text {tot }}\right)$, where the uncertainties account for lowest and higher order hadronic, and other contributions, respectively. The result deviates from the experimental value, $a_{\mu}^{\exp }=11659209.1 \pm$ $5.4 \pm 3.3$ [17], by $26.8 \pm 7.6(3.5 \sigma)$. A compilation of recent SM predictions for $a_{\mu}$ compared with the experimental result is given in Fig. 1 .

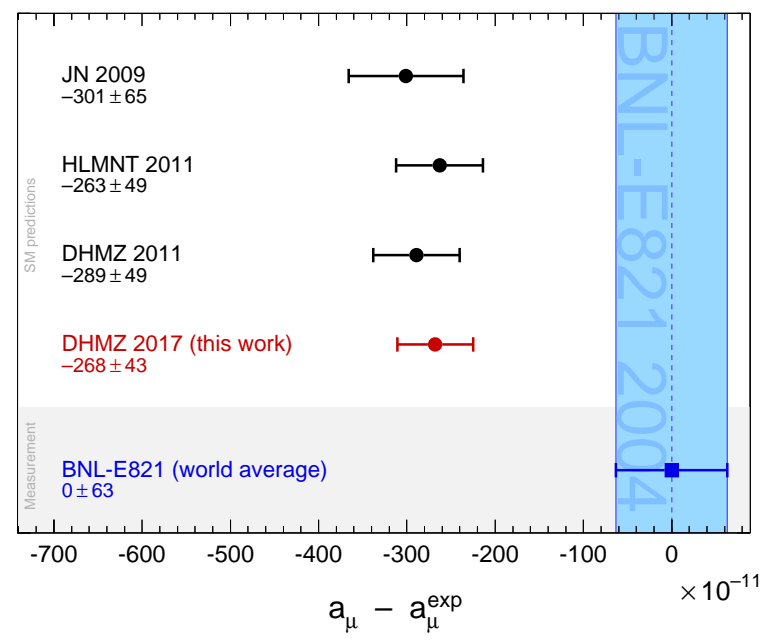

Figure 1. Compilation of recent results for $a_{\mu}^{\mathrm{SM}}$ (in units of $10^{-11}$ ), subtracted by the central value of the experimental average. The shaded vertical band indicates the experimental uncertainty. The representative SM predictions are taken from JN 2009 [18], HLMNT 2011 [19], DHMZ 2011 [3], and DHMZ 2017 [1].

Similar reevaluation is also performed for $\Delta \alpha_{\text {had }}\left(m_{Z}^{2}\right)$ with the result of $(275.3 \pm 0.9) \cdot 10^{-4}[1]$. The uncertainty is dominated by data systematic effects $\left(0.7 \cdot 10^{-4}\right)$ and the uncertainty in the QCD prediction $\left(0.6 \cdot 10^{-4}\right)$.

\section{Summary}

Using newest available $e^{+} e^{-} \rightarrow$ hadrons cross-section data, the uncertainty of $0.5 \%$ on $a_{\mu}^{\text {had,LO }}$ is now reduced to about half the current uncertainty of the $a_{\mu}$ measurement, and has improved by more than a factor of two during the last thirteen years. The discrepancy between measurement and complete Standard Model prediction remains at a non-conclusive 3.5 $\sigma$ level. The forthcoming experiments at Fermilab [20] and J-PARC [21], aiming at up to four times better ultimate precision, have the potential to clarify the situation. 


\section{References}

[1] M. Davier, A. Hoecker, B. Malaescu, and Z. Zhang, arXiv:1706.09436 [hep-ph].

[2] M. Davier, A. Hoecker, B. Malaescu, C.Z. Yuan, and Z. Zhang, Eur. Phys. J. C 66 (2010) 1 [arXiv:0908.4300 [hep-ph]].

[3] M. Davier, A. Hoecker, B. Malaescu, and Z. Zhang, Eur. Phys. J. C 71 (2011) 1515, erratum-ibid, C 72 (2012) 1874 [arXiv:1010.4180 [hep-ph]].

[4] KLOE Collaboration, Phys. Lett. B 720, 336 (2013) [arXiv:1212.4524 [hep-ex]].

[5] BESIII Collaboration, Phys. Lett. B 753, 629 (2015) [arXiv:1507.08188 [hep-ex]].

[6] BABAR Collaboration, Phys. Rev. D 85, 112009 (2012) [arXiv:1201.5677 [hep-ex]].

[7] CMD3 Collaboration, Phys. Lett. B 768, 345 (2017) [arXiv:1612.04483 [hep-ex]].

[8] BABAR Collaboration, arXiv:1709.01171 [hep-ex].

[9] BABAR Collaboration, Phys. Rev. D 88, 032013 (2013) [arXiv:1306.3600 [hep-ex]].

[10] SND Collaboration, Phys. Rev. D 94, 112006 (2016) [arXiv:1608.08757 [hep-ex]].

[11] BABAR Collaboration, Phys. Rev. D 89, 092002 (2014) [arXiv:1403.7593 [hep-ex]].

[12] CMD3 Collaboration, Phys. Lett. B 760, 314 (2016) [arXiv:1604.02981 [hep-ex]].

[13] A. Kurz, T. Liu, P. Marquard, and M. Steinhauser, Phys. Lett. B 734, 144 (2014) [arXiv:1403.6400 [hep-ph]].

[14] J. Prades, E. de Rafael, and A. Vainshtein, Ser. Direct. High Energy Phys. 20, 303 (2009) [arXiv:0901.0306 [hep-ph]].

[15] T. Aoyama, M. Hayakawa, T. Kinoshita, and M. Nio, Phys. Rev. Lett. 109, 111808 (2012) [arXiv:1205.5370 [hep-ph]].

[16] C. Gnerdiger, D. Stöckinger, and H. Stöckinger-Kim, Phys. Rev. D 88, 053005 (2013) [arXiv:1306.5546 [hep-ph]].

[17] Muon $g-2$ Collaboration, Phys. Rev. D 73, 072003 (2006) [hep-ex/0602035].

[18] F. Jegerlehner and A. Nyffeler, Phys. Rept. 477, 1 (2009) [arXiv:0902.3360 [hep-ph]].

[19] K. Hagiwara et al., J. Phys. G 38, 085003 (2011) [arXiv:1105.3149 [hep-ph]].

[20] Muon $g-2$ Collaboration, FERMILAB-DESIGN-2014-02, arXiv:1501.06858 [physics.ins-det].

[21] E34 muon $g-2 / E D M$ experiment at J-PARC, http://g-2 . kek. jp/portal/index.html. 